\title{
Female versus male: Rumex thyrsiflorus Fingerh. under in vitro conditions. Does sex influence in vitro morphogenesis?
}

\author{
Halina Ślesak $^{1} \cdot$ Katarzyna Dziedzic $^{1} \cdot$ Dagmara Kwolek $^{1} \cdot$ Magdalena Cygan $^{1}$. \\ Patryk Mizia $^{1} \cdot$ Paweł Olejniczak $^{2} \cdot$ Andrzej J. Joachimiak $^{1}$
}

Received: 8 September 2016 / Accepted: 24 February 2017 / Published online: 14 March 2017

(c) The Author(s) 2017. This article is published with open access at Springerlink.com

\begin{abstract}
Rumex thyrsiflorus Fingerh. is a dioecious plant with polymorphic sex chromosomes (XX in females, $\mathrm{XY}_{1} \mathrm{Y}_{2}$ in males). This species is an interesting plant for study on the structure and function of sex chromosomes, the sex ratio, and is also a valuable source of bioactive constituents. A procedure for the micropropagation of $R$. thyrsiflorus from hypocotyl explants, which can be useful for analyses of sex-specific morphogenetic reactions under in vitro conditions, was developed. The molecular analysis, based on genetic sex markers, allowed the sex of explants to be determined in order to create male or female in vitro cultures. Regeneration primarily occurred via indirect adventitious shoot formation as well as via somatic embryogenesis, which was confirmed by histological and scanning electron microscope (SEM) analyses. The highest frequency of explants that revealed a morphogenetic response with the highest number of regenerated adventitious shoot buds (average 11.5 shoots per explant) was obtained on a Murashige and Skoog (MS) medium supplemented with $2.27 \mu \mathrm{M}$ thidiazuron (TDZ). Molecular analysis revealed a female-biased sex ratio under these conditions. The relationship between the sex of the explants and their morphogenetic potential was studied. The efficiency of morphogenesis varied between the two sexes and depended on the ratio of auxin and cytokinin in the medium. The germination pattern of male and female seeds under in vitro conditions was also analysed. No differences in germination time
\end{abstract}

Halina Ślesak

halina.slesak@uj.edu.pl

1 Department of Plant Cytology and Embryology, Jagiellonian University, 9 Gronostajowa Str., 30-387 Cracow, Poland

2 Institute of Nature Conservation PAS, 33 Mickiewicza Ave., 31-120 Cracow, Poland between male and female seeds and in terms of male and female seedling length were observed, which indicated that there was no competition between the sexes during seed germination when grown under uniform growth conditions.

Keywords Organogenesis - Somatic embryogenesis · Histological analysis · Scanning electron microscopy · Sex ratio $\cdot$ Genetic sex marker

\section{Introduction}

Rumex thyrsiflorus Fingerh. (thyrse sorrel) is a dioecious plant with polymorphic sex chromosomes. It belongs to the family Polygonaceae, which are rich sources of bioactive constituents that have a wide range of medicinal properties (Orbán-Gyapai et al. 2015). The phytopreparations that can be isolated from $R$. thyrsiflorus exhibit, e.g., haemostatic, anti-inflammatory, antiscorbutic, and antiseptic activities (Litvinenko and Muzychkina 2008). Antiproliferative activities against cancer cells were reported for this Rumex species (Lajter et al. 2013). Rumex thyrsiflorus is closely related to $R$. acetosa. The chromosome constitution of females in both species is $2 \mathrm{n}=12 \mathrm{~A}+\mathrm{XX}$ and in males is $2 \mathrm{n}=12 \mathrm{~A}+\mathrm{XY}_{1} \mathrm{Y}_{2}$ (Żuk 1963). These species are attractive subjects for studies on chromosomes, sex chromatin, and sex ratio, as well as for comparing the primary (seeds) and the secondary (populations) sex ratio (Rychlewski and Zarzycki 1986; Błocka-Wandas et al. 2007; GrabowskaJoachimiak et al. 2012).

Biased sex ratios in populations have been observed in many dioecious plants. According to Hardy (2002), a negative frequency-dependent selection should result in equal sex ratios in equilibrium populations of dioecious flowering plants, however deviations from equality have also 
been reported. This phenomenon could be explained by ecological and genetic factors, nevertheless, the mechanisms involved are not well understood (Pickup and Barrett 2013). According to Korpelainen (2002), in the case of chromosomal sex determination the primary sex ratio $1: 1$ is expected. However, the number of males per females at sexual maturity (operational sex ratio) may be biased because of differences between the sexes in germination, flowering frequency, mortality, vegetative vigour, or because of a genetic mechanism that distorts the sex ratio.

A compilation of plant sex ratios in 243 species of flowering plants representing 123 genera and 61 families, conducted by Field et al. (2012), revealed significantly biased ratios in $50.2 \%$ of the analysed species with a male bias much more common than female bias $(31.3 \%$ and $18.9 \%$, respectively). In dioecious plants, females may incur higher reproductive costs than males because they produce the seeds, and as a result the reduced growth, higher mortality rates, delayed flowering, or a lower frequency of reproduction could be observed (Stehlik and Barret 2005). Early maturation of male individuals is common in dioecious plants, probably because of their lower costs of reproduction compared to females (Obeso 2002; Ueno et al. 2006). As a result of earlier reproduction of males, there could be a higher number of males in young populations (Ueno et al. 2007 and references therein). In most cases, the data on sex ratios focuses on flowering plants, because it is difficult to evaluate the sex of non-reproductive individuals without genetic sex markers, and one cannot exclude the sex-related differences in seed viability (Stehlik and Barrett 2005; Pickup and Barrett 2013). For these reasons, studies on the primary sex ratios and biased sex-ratio during the early developmental stages are limited (Korpelainen 2002, Stehlik and Barret 2005).

Female-biased sex ratios are often observed in species with heteromorphic sex chromosomes (Lloyd 1974), thus suggesting a relationship between male and female vitality and the sex-determination system. Polymerase chain reaction (PCR)-based studies on gender among the seeds of $R$. thyrsiflorus and $R$. acetosa, carried out using DNA markers located on Y chromosomes (Kwolek and Joachimiak 2011) revealed female-biased primary sex ratios in these species. In experimental populations of $R$. acetosa and $R$. thyrsiflorus, the sex ratios among adult individuals were more female-biased than among seeds and seedlings, which was determined cytologically (Rychlewski and Zarzycki 1986) and by using of male-specific genetic markers (Korpelainen 2002).

According to Stehlik and Barrett (2005), the femalebiased seed or seedling sex ratios in the subgenus Acetosa, which includes $R$. thyrsiflorus, $R$. nivalis, and $R$. acetosa, and the postparental care male-biased mortality in $R$. thyrsiflorus (Rychlewski and Zarzycki 1986) and $R$. nivalis
(Stehlik and Barrett 2005), revealed the possibility that there may be observed a functional association between the degree of sex-chromosome differentiation, degeneration of Y-chromosome, and sex ratio variation in dioecious species with a chromosomal sex determination system. Additionally, studies on the viability of the sexes of $R$. nivalis showed that more stressful conditions may lead to higher male mortality (Stehlik and Barrett 2005). All of these results on the "female predominance" in Rumex species raised the question about the behaviour of female and male explants under in vitro conditions.

The objectives of this study were to develop a regeneration procedure for $R$. thyrsiflorus under in vitro conditions and to answer the following questions: (1) Are there sexrelated differences in the rate of seed germination and the size of seedlings? Do male and female seeds have different germination patterns? and (2) Does sex influence morphogenesis under in vitro conditions? Is there a relationship between the sex of explants, their ability to undergo morphogenesis, and the type of plant growth regulators that are exogenously supplied to culture medium?

\section{Materials and methods}

\section{Plant material and culture conditions}

Seeds of $R$. thyrsiflorus Fingerh. (PlantiCo, Poland) were surface-disinfected in $70 \%(\mathrm{v} / \mathrm{v})$ ethanol for $1 \mathrm{~min}$ and then immersed in $50 \%(\mathrm{v} / \mathrm{v})$ bleach solution $(<5 \%$ sodium hypochlorite) (Procter \& Gamble, Poland) for $12 \mathrm{~min}$, followed by three rinses in sterile distilled water. An in vitro experiment was conducted in order to investigate whether female and male seeds differ in their germination ability, and to estimate the relationship between sex and the length of seedlings. Samples of 150 randomly selected seeds were germinated on autoclaved moist filter paper (qualitative, basis weight $\left.65 \mathrm{~g} / \mathrm{m}^{2}\right)$ in Petri dishes $(90 \mathrm{~mm})$ at $26 \pm 3^{\circ} \mathrm{C}$ under a $16 \mathrm{~h}$ photoperiod (cool-white fluorescent tubes,

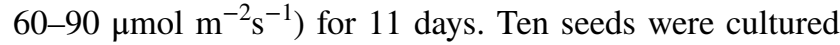
per Petri dish. The seeds were monitored on a daily basis in order to record the exact day of germination. The following traits were measured for each seed: the number of days from sowing to appearance of the roots and cotyledons, and size of the seedlings 11 days after sowing. The length of each seedling was measured from root tip to shoot tip.

Another sample of randomly selected seeds (ap. 350) was used for the micropropagation experiment in which hypocotyls (ca $5 \mathrm{~mm}$ ) that had been isolated from 11 dayold seedlings were used as explants (these had been selected as the most promising in preliminary experiments, data not shown). Hypocotyls were cultured on Murashige and Skoog (MS) medium (Murashige and Skoog 1962) 
supplemented with different concentrations of sucrose, 2,4-dichlorophenoxyacetic acid (2,4-D), benzylaminopurine (BA) and thidiazuron (TDZ) (Table 1). The media were solidified with $0.8 \%$ (w/v) agar (MP Biomedicals). The cultures were incubated at $26 \pm 3{ }^{\circ} \mathrm{C}$ under a $16 \mathrm{~h}$ photoperiod

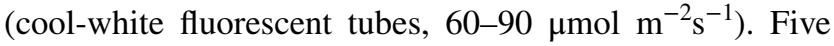
explants were cultured per Petri dish $(60 \mathrm{~mm}, 10 \mathrm{ml}$ of MS medium) and at least ten replicates (Petri dishes) were used for each treatment.

The regeneration efficiency was evaluated by calculating the frequency $(\%)$ of the explants that revealed a morphogenetic response, and the number of regenerated adventitious shoot buds per explant. $98 \%$ of the regenerated shoots were rooted in a 100-ml glass vessel with $25 \mathrm{ml}$ of MS medium supplemented with $2 \%$ sucrose and $2.46 \mu \mathrm{M}$ indole-3-butyric acid (IBA). Rooted plantlets were acclimatized in a phytotron chamber $\left(24^{\circ} \mathrm{C}, 16 / 8 \mathrm{~h}\right.$ photoperiod) and then planted in field conditions.

\section{Sex ratio analysis}

In order to determine the sex of the hypocotyls that were used as explants for in vitro culture, the cotyledons and roots that had been isolated from the same germinated seedling from which the hypocotyl was excised were used for DNA isolation. Genomic DNA was extracted using the hexadecyltrimethylammonium bromide (CTAB) method (Gawal and Jarret 1991) with modifications (Kwolek and Joachimiak 2011). PCR-based methods that involved the DNA markers that were located on the Y chromosomes were used to identify the male seedlings and cultured explants. The following primers, developed by Korpelainen (2002), RAY-F (5'-ACTCGAATGTAAGCATTTGGTCCT A-3') and RAY-R (5'-ACTACACGATTGTCCATAAAG TGGA-3'), were used to amplify the male-specific RAYSI sequence, that is present on the Y chromosomes of $R$. acetosa and its close relatives (Navajas-Peréz et al. 2006). Additionally, UGR08-F (5'-CCAATTGGTCTCAACTAG AACA-3') and UGR08-R (5'-TGTTATAGGTTTTGGACT GCCA-3'), primers specific for the male-specific repetitive sequence RAYSII (Mariotti et al. 2009) were used. Amplification with the primers R730-A (5'-CTCGGACCAATT ATCTCAT-3') and R730-B (5'-CATTATTTGGGAGCC GAT-3') (Navajas-Peréz et al. 2005) was carried out to verify the template DNA quality. These primers amplify the repetitive RAE730 sequence that is located on the Rumex autosomes. The amplification reaction was carried out in a T100 Thermal Cycler (BioRad) (for details of all of the reaction mixtures and programs see Ślesak et al. 2015). The PCR products were separated in $1 \%(\mathrm{w} / \mathrm{v})$ agarose gel with a 1xTBE buffer and stained using Simply Safe (EURx). The GeneRuler 100 bp PLUS DNA Ladder (Thermo Scientific) was used as the molecular standard.

\section{Histological and scanning electron microscopy (SEM) analyses}

For the histological analysis, the material was prepared for embedding the tissues in Technovit 7100 synthetic resin (2-hydroxyethyl-methacrylate) (Heraeus Kulzer). The explants were fixed in $5 \%$ buffered $(0.1 \mathrm{M}$ phosphate buffer, $\mathrm{pH}$ 7.2) glutaraldehyde at room temperature for $24 \mathrm{~h}$, rinsed four times in the same phosphate buffer (PBS) followed by dehydration in a graded ethanol series $(10,30$, 50,70 , and $90 \%$ ) for $15 \mathrm{~min}$ at each concentration and kept overnight in absolute ethanol. Samples were then infiltrated in a mixture of absolute ethanol and Technovit $(1 \mathrm{~h}$ at each proportion: $3: 1,1: 1,1: 3 ; \mathrm{v} / \mathrm{v})$ and stored for $12 \mathrm{~h}$ in pure Technovit. The resin was polymerised by adding a hardener. The material was sectioned to $5 \mu \mathrm{m}$ using a rotary microtome (Microm, Adamas Instrumenten) and stained using periodic acid Schiff (PAS) and naphthol blue black (NBB) double staining as described by Ślesak et al. (2014).

In the PAS reaction, the total carbohydrates of insoluble polysaccharides (e.g. starch and cellulose) stain magenta to purplish red. NBB is useful in staining total proteins and nucleic acids. The material was mounted in Entellan ()$_{\text {syn- }}$ thetic resin (Merck, Germany). Microscopic sections were photographed using a Nikon DS-Fi2 with NIS-Elements D 4.00.00 4.0 software.
Table 1 The media used in the experiment, which were based on MS (Murashige and Skoog 1962), supplemented with different plant growth regulators at different concentrations and the frequency of morphogenesis

\begin{tabular}{lllll}
\hline Medium & Auxin $(\mu \mathrm{M})$ & Cytokinin $(\mu \mathrm{M})$ & Sucrose $(\%)$ & $\begin{array}{l}\text { No. of explants used; } \% \\
\text { of explants with morpho- } \\
\text { genesis }\end{array}$ \\
\hline A & 2,4-D (4.52) & BA (2.22) & 5 & $49 ; 20.4$ \\
B & 2,4-D (4.52) & BA (8.88) & 5 & $50 ; 16$ \\
C & 2,4-D (9.05) & BA (2.22) & 5 & $48 ; 16.7$ \\
D & 2,4-D (9.05) & BA (8.88) & 5 & $50 ; 10$ \\
E & & TDZ (2.27) & 3 & $69 ; 26.1$ \\
\hline
\end{tabular}

2,4-D 2,4-dichlorophenoxyacetic acid, $B A$ benzylaminopurine, $T D Z$ thidiazuron 
For SEM analysis, the material was pre-fixed in 5\% glutaraldehyde (0.1 M phosphate buffer, $\mathrm{pH}$ 7.2) for $24 \mathrm{~h}$ at room temperature. Samples were then rinsed in a PBS and dehydrated through a graded ethanol series $(10,30,50$, 70, 90, and $100 \%$ ). Samples were then dried using a $\mathrm{CO}_{2}$ critical point drying system (Leica EM CPD300), sputtercoated with gold (Jeol JFC-1100 E ion-sputtering system), and observed using a SEM Phenom G2 pro. Microscopic sections were photographed using a Navigation Camera with a 20-120× magnification.

\section{Data analysis}

All statistical data analyses were performed using SPSS version 24.0 (IBM Corp. 2016). The length of time for germination of seeds was measured as the number of days from sowing to the appearance of roots and cotyledons (both were assessed separately). The difference in the germination time between the sexes was tested using a generalised linear model with a Poisson log linear model and with sex as a factor. The difference between the sexes in terms of seedling length measured on the 11th day after sowing were checked using the $t$ test.

The effect of the culture medium on the morphogenesis of explants of the different sexes was analysed in a generalised linear model. In this analysis, a binary logistic model was used and the type of culture medium and sex were defined as the factors. Sex ratio, i.e. the male to female ratio was calculated according to the formula 1:F/M (Rychlewski and Zarzycki 1975).

\section{Results}

\section{Molecular analysis}

Amplification of RAYSI sequence with the use of RAY-F and RAY-R primers showed a 930 bp product (Fig. 1a) in all males. A DNA band of similar length was also obtained in some females, but there was a difference in the intensity of the amplified bands between the sexes. The amplification of the RAYSII sequence with the primers UGR08-F and UGR08-R resulted in a product about $700 \mathrm{bp}$ that was visible in all of the analysed male plants. An additional product around $600 \mathrm{bp}$ in size was also present (Fig. 1b). Amplification with R730-A and R730-B primers, was carried out to verify the template DNA quality. In this case, the PCR product was obtained for all of the analysed explants, which confirmed the good quality of DNA used for gender determination (Fig. 1c).

\section{Seed germination and growth of seedlings: female versus male}

Molecular analysis revealed that among the 150 seedlings that were generated for this study: $31.3 \% \quad(n=47)$ were male and $68.7 \%(n=103)$ were female (sex ratio $\mathrm{M}: \mathrm{F}=1: 2.19)$. The germination of the seeds was measured as the number of days from sowing to the appearance of the roots (Fig. 2a) and cotyledons (Fig. 2b) in male and female seeds. The highest frequency of root induction was observed on day three of culture (60 female seeds, $58.3 \%$, 28 male seeds, 59.6\%) (Fig. 2a). The highest frequency of

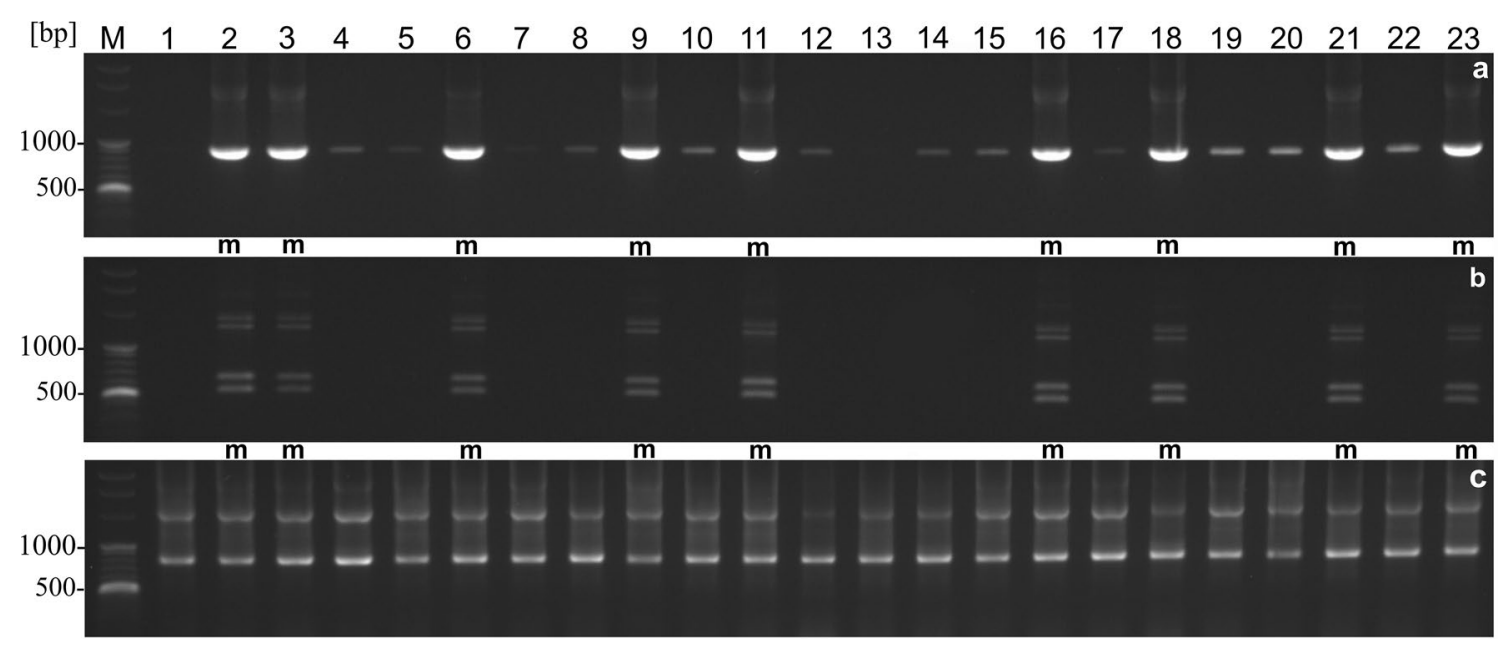

Fig. 1 PCR products in Rumex thyrsiflorus explants cultured in vitro (1-23): RAY-F and RAY-R primers (a), UGR08-F and UGR08-R primers (b), R730-A and R730-B primers (c). $m$ male hypocotyls, M-100 bp molecular weight marker 

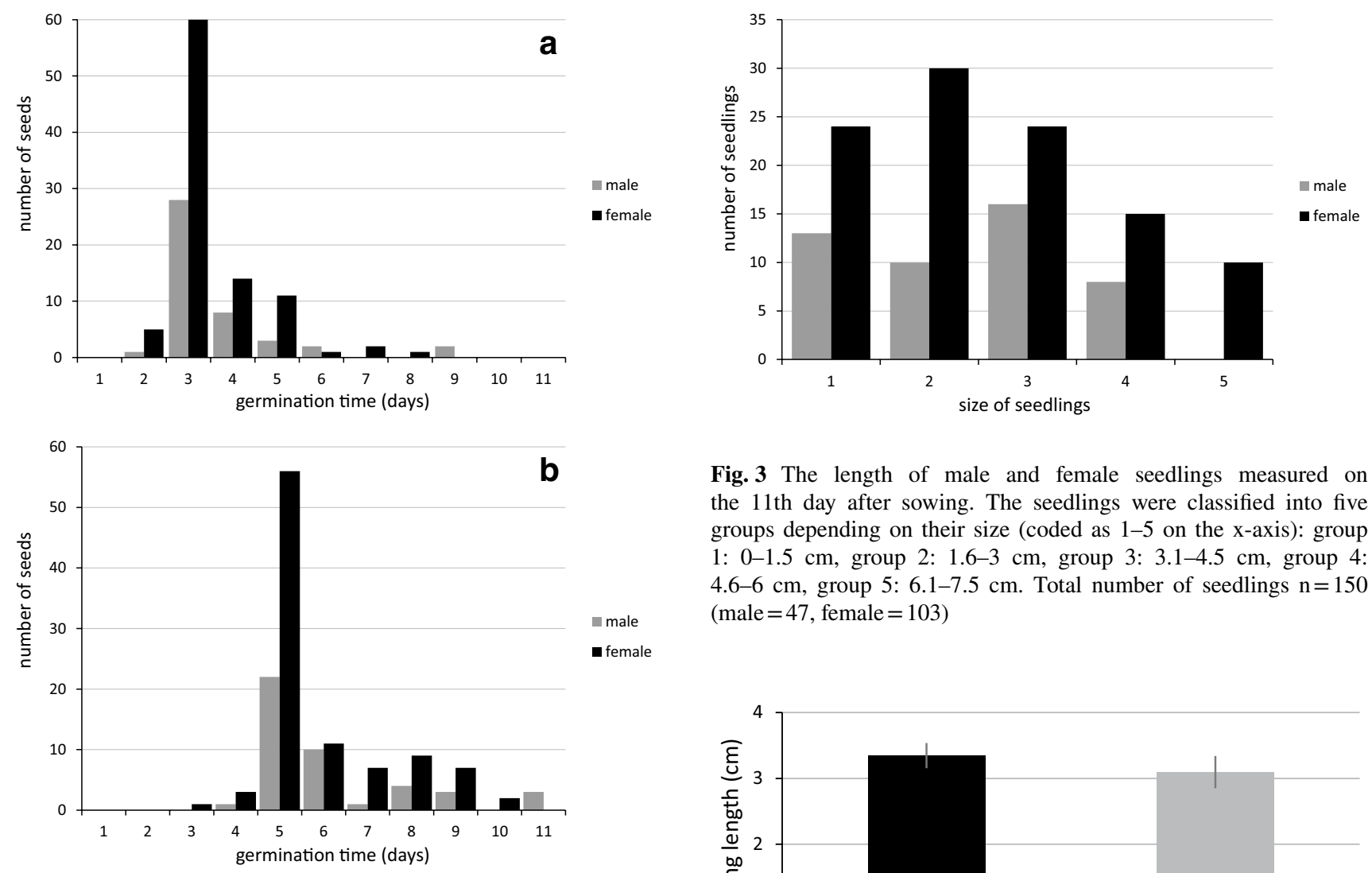

Fig. 3 The length of male and female seedlings measured on the 11th day after sowing. The seedlings were classified into five groups depending on their size (coded as $1-5$ on the $\mathrm{x}$-axis): group 1: $0-1.5 \mathrm{~cm}$, group 2: $1.6-3 \mathrm{~cm}$, group $3: 3.1-4.5 \mathrm{~cm}$, group 4 : $4.6-6 \mathrm{~cm}$, group 5: $6.1-7.5 \mathrm{~cm}$. Total number of seedlings $\mathrm{n}=150$ $($ male $=47$, female $=103)$

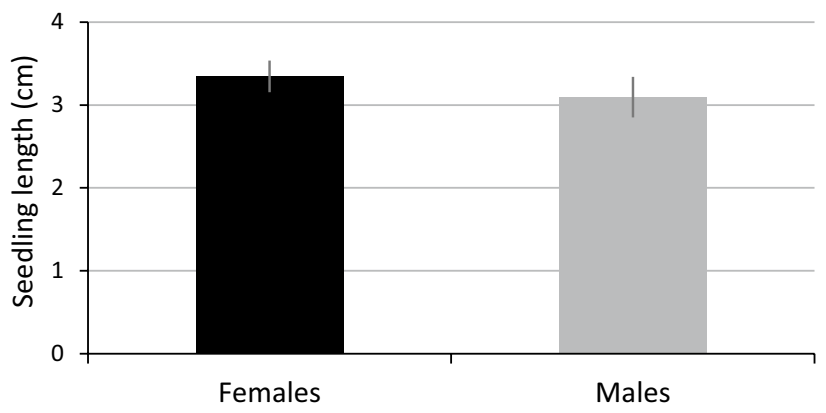

Fig. 2 Germination of male and female seeds measured as the number of days that elapsed from sowing to the appearance of roots (a) and cotyledons (b). Total number of seeds $n=150$ (male $=47$, female $=103$ )

cotyledon development induction was visible on day five of culture (56 female seeds, $54.4 \%, 22$ male seeds, $46.8 \%$ ) (Fig. 2b). No differences in germination time between the sexes were found both when the roots appeared (Wald $\left.\chi^{2}=0.275 ; \mathrm{df}=1 ; \mathrm{p}=0.600\right)$ and the appearance of cotyledons (Wald $\chi^{2}=0.545 ; \mathrm{df}=1 ; \mathrm{p}=0.460$ ) were considered.

The length of the seedlings was measured on the 11th day after sowing. The seedlings were classified into five groups depending on their size: group 1: $0-1.5 \mathrm{~cm}$, group 2: $1.6-3 \mathrm{~cm}$, group 3: $3.1-4.5 \mathrm{~cm}$, group $4: 4.6-6 \mathrm{~cm}$, and group 5: $6.1-7.5 \mathrm{~cm}$ (Fig. 3). Among the seedlings in group 5 (the longest seedlings), only female seedlings were visible (10 female seedlings, 9.7\%). Statistical analysis revealed that the differences between the sexes in terms of seedling length were non-significant $(t$ test; $\mathrm{t}=0.762$; $\mathrm{df}=136 ; \mathrm{p}=0.447)$ (Fig. 4).

\section{Morphogenesis in vitro}

The first complete micropropagation protocol from hypocotyl explants was developed for $R$. thyrsiflorus during

Fig. 4 Differences between the sexes in terms of seedling length measured on the 11th day after sowing. Means \pm SE are denoted

this study. The induction of callus was observed on all explants 2 weeks after initiation of culture, irrespective of their sex or the culture medium. Callus was observed on the cut surface initially (Fig. 5a) and subsequently on the whole explant. The first signs of a morphogenetic response were visible on medium supplemented with $2.27 \mu \mathrm{M}$ TDZ (medium E in Table 1) 21 days after culture initiation. The regeneration primarily occurred via indirect organogenesis (formation of adventitious shoots via callus). Several adventitious shoots surrounded by callus were visible (Fig. 5b). Somatic embryos or somatic embryo-like structures were also observed on medium supplemented with $9.05 \mu \mathrm{M} 2,4-\mathrm{D}$ and $8.88 \mu \mathrm{M}$ BA (medium D in Table 1).

SEM analysis (Fig. 5c-f) of morphogenic callus indicated adventitious shoot formation. Secondary organogenesis was also observed (Fig. 5c). There were typical looking glandular cells on the surface of the adventitious shoots (Fig. 5d). In some cases, there were visible structures that were similar to malformed adventitious shoots 

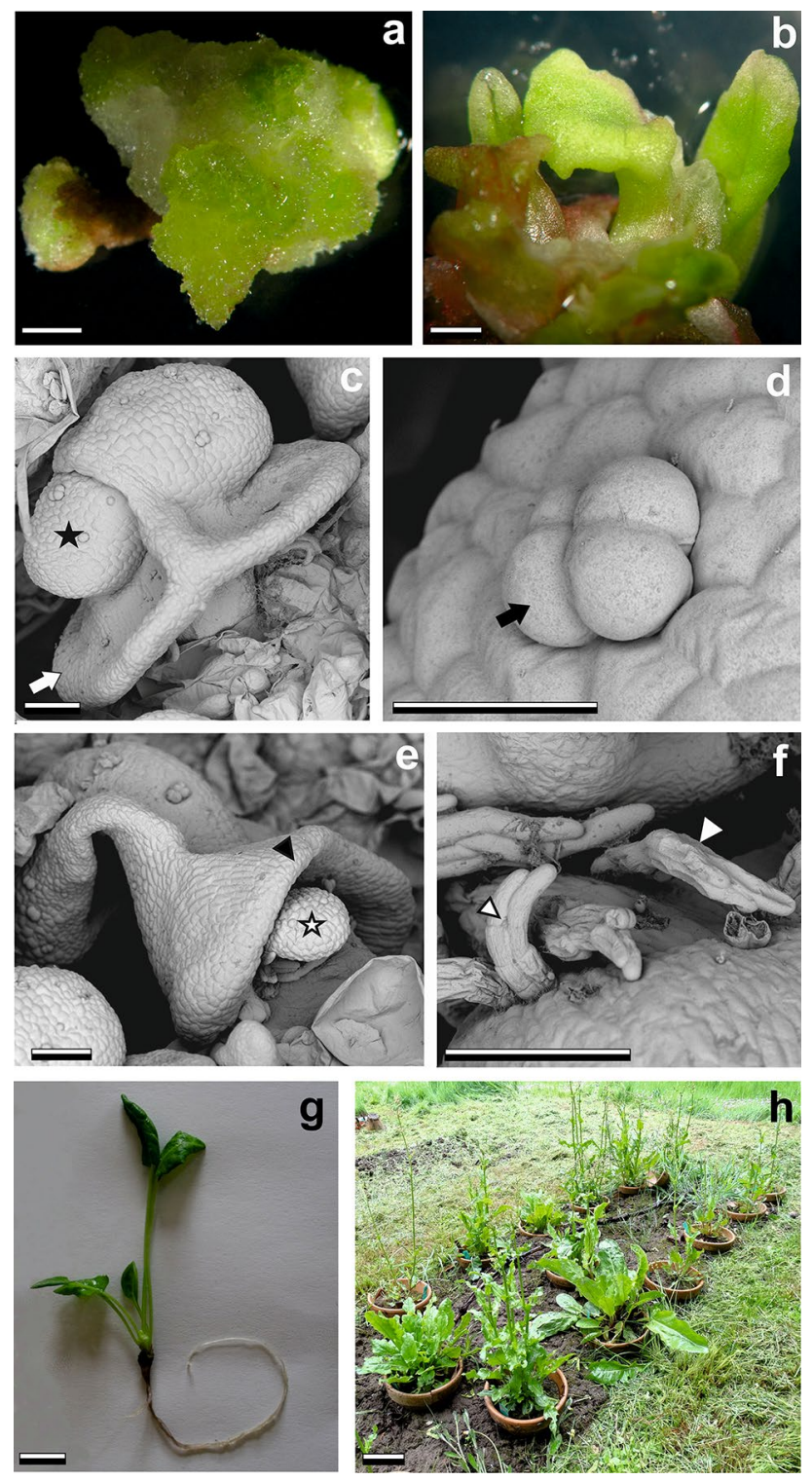

Fig. 5 Adventitious shoot regeneration and SEM images $(\mathbf{c}-\mathbf{f})$ of a morphogenic callus cultured on MS $+2.27 \mu \mathrm{M}$ TDZ. Callus induction on a hypocotyl after 28 days of culture (a); adventitious shoot regeneration (6 weeks of culture) (b); note the malformed regenerated plantlet (white arrow) and secondary organogenesis (black asterisk) (c); visible typical looking glandular cells (black arrow) on the surface of adventitious shoots (d) (18 weeks of culture); malformed plantlet or shoot with a visible apical meristem (white asterisk) surrounded by connate leaves (black arrowhead) (e); multicellular trichome-like structures (white arrowheads) were also present (f); regenerated plantlet rooted (g) and acclimated to field conditions (h). Bars $2 \mathrm{~mm}(\mathbf{a}, \mathbf{b}), 100 \mu \mathrm{m}(\mathbf{c}, \mathbf{e}, \mathbf{f}), 30 \mu \mathrm{m}(\mathbf{d}), 10 \mathrm{~mm}(\mathbf{g}), 100 \mathrm{~mm}$ (h)

with an apical meristem, which were surrounded by connate leaves (Fig. 5e) and multicellular trichome-like structures (Fig. 5f). The regenerated shoots were transferred to a rooting medium, and the rooted plantlets (Fig. 5g) were acclimatized to field conditions (Fig. 5h).
The highest frequency of explants that revealed a morphogenetic response (26.1\%) with the highest number of regenerated adventitious shoot buds (average 11.5 shoots per explant) was observed on medium supplemented with $2.27 \mu \mathrm{M}$ TDZ (medium E in Table 1). The shoot that regenerated on this medium were acclimatized to ex vitro conditions with $>90 \%$ survival rate.

\section{Histological analysis}

Cross sections of hypocotyls after 1 week in culture revealed numerous starch grains inside the highly vacuolated callus cells in the cortex layer and inside the stele cells (Fig. 6a). An accumulation of starch grains inside the callus cells was observed during the first 4 weeks after culture initiation. The heterogeneous callus consisted of cells that varied in shape, size, and degree of vacuolisation. Large, highly vacuolated dividing callus cells were loosely packed and filled with starch grains visible around the nucleus (Fig. 6b). Meristematic centres that were composed of isodiametric, small cells with a dense cytoplasm were located on the surface and in the internal region of the callus (Fig. 6c).

After 4 weeks, there were visible adventitious shoots with leaf primordia and an apical meristem, which had regenerated indirectly (via callus) (Fig. 6d). A longitudinal section of callus that was obtained on medium D (Table 1) revealed globular (Fig. 6e) and heart-stage somatic embryos (Fig. 6f) that were visible on the surface layer of cultured explants.

\section{Morphogenesis in vitro: female versus male}

Molecular analysis based on genetic sex markers revealed a female-biased sex ratio (M:F=1:1.96) among the explants. The relationship between the sex of the explants and their morphogenetic potential was analysed. The efficiency of morphogenesis varied in the sexes and depended on the ratio of auxin and cytokinin in the culture medium (Fig. 7a-e). A higher efficiency in morphogenetic reaction in the female explants compared to the male explants was observed on medium A and D (Table 1). A molecular analysis of all explants cultured on medium A revealed a sex ratio of $\mathrm{M}: \mathrm{F}=1: 1.22(44.9 \%$ male and $55.1 \%$ were female). A sex analysis of the explants with morphogenetic potential revealed an increased participation of female hypocotyls and a sex ratio of $\mathrm{M}: \mathrm{F}=1: 2.33$ (30\% were male and $70 \%$ were female) compare to non-morphogenic explants (M:F $=1: 1.05,48.7 \%$ were male and $51.3 \%$ were female) (Fig. 7a). Similarly, on medium D, the sex ratio among the explants was $\mathrm{M}: \mathrm{F}=1: 1.38$ (42\% were male and 58\% were female). Among the explants that revealed a morphogenetic reaction on this medium, the sex ratio 
Fig. 6 Histological sections of explants cultured on MS +2.27 $\mu \mathrm{M}$ TDZ $(\mathbf{a}, \mathbf{d})$ and MS +9.05 $\mu \mathrm{M} 2,4-\mathrm{D}+8.88 \mu \mathrm{M}$ BA (b, c, e, f). Cross section of a hypocotyl after 1 week of culture (a), numerous starch grains inside highly vacuolated callus cells are visible in the cortex layer (cor) and inside the stele cells (black asterisk). Heterogeneous callus tissue composed of cells that vary in shape, size and degree of vacuolisation $(\mathbf{b}, \mathbf{c})$; large, highly vacuolated dividing callus cells that are loosely attached each other and starch grains (black arrows) around the nucleus are visible (b) and meristematic centres that are composed of small, isodiametric cells with dense cytoplasm are visible deep inside the callus (c). Cross section of a regenerated adventitious shoot (4 weeks of culture) with leaf primordia (white arrows) and an apical meristem (black arrowhead) (d). Longitudinal section of a callus with a visible globular somatic embryo (e) and heart-stage somatic embryo (f) (7 weeks of culture). All sections (a-f) were stained using periodic acid Schiff (PAS) and naphthol blue black (NBB) double staining. Bars $200 \mu \mathrm{m}$ (a), $50 \mu \mathrm{m}(\mathbf{b}, \mathbf{d}, \mathbf{e}), 100 \mu \mathrm{m}(\mathbf{c}, \mathbf{f})$
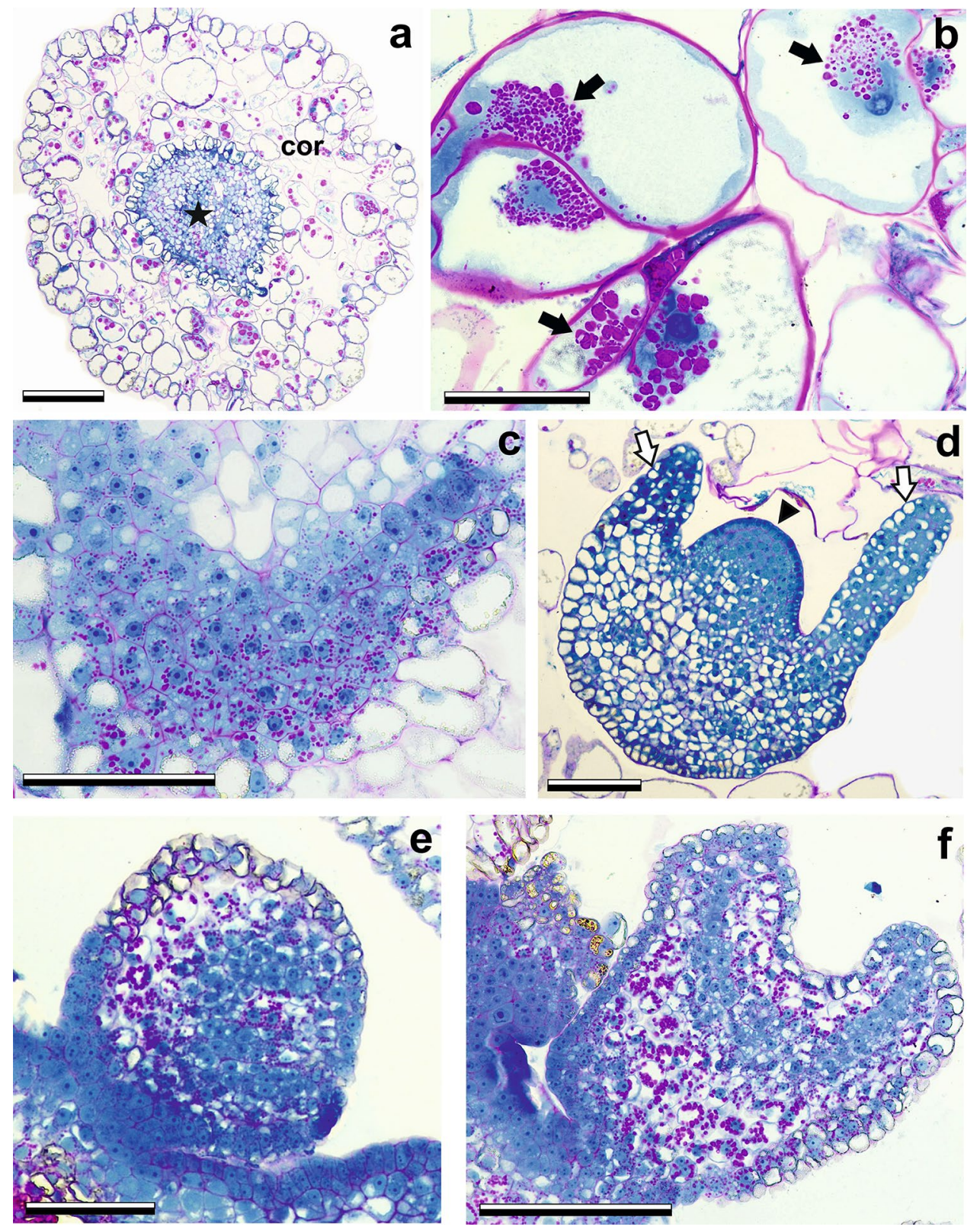

was more female-biased at $\mathrm{M}: \mathrm{F}=1: 4$ (only $20 \%$ were male and $80 \%$ were female) compare to non-morphogenic explants (M:F $=1: 1.25,44.4 \%$ were male and $55.6 \%$ were female) (Fig. 7d). The opposite reaction was observed on medium B (Table 1) where an increase in the participation of male explants was evident with morphogenetic potential $(\mathrm{M}: \mathrm{F}=1: 1.67 ; 37.5 \%$ of males among the morphogenetic explants) compared to non-morphogenic explants (M:F=1:2.82; 26.2\% of males) (Fig. 7b). Although the described tendency was observed on individual medium, the relationship between the type of culture medium and the sex of the explants in terms of morphogenesis was not statistically significant (Table 2).

\section{Discussion}

Sex ratio, which has an impact on population structure, mate competition, and reproductive fitness, is a key factor in evolutionary biology. Investigations of sex ratios in plants are complicated because of the difficulty in distinguishing the sexes of individuals during the juvenile phases because of weakly developed secondary sex characteristics (Stehlik et al. 2007). Only a few studies of sex ratios in wild plant populations using sex-specific molecular markers have included non-flowering individuals. During our analysis, we confirmed the usefulness of the RAY-F and RAY-R primers (Korpelainen 2002). These primers, which amplify RAYSI, the male-specific sequence that was present on the 
Fig. 7 The relationship between sex and morphogenesis on media that are coded as a-e in Table 1. The first bar on each graph shows the sex ratio among explants that did not revealed a morphogenic response (non-morphogenic) on a medium and the second bar represents the sex ratio among the explants that revealed a morphogenetic response (morphogenic) on this medium
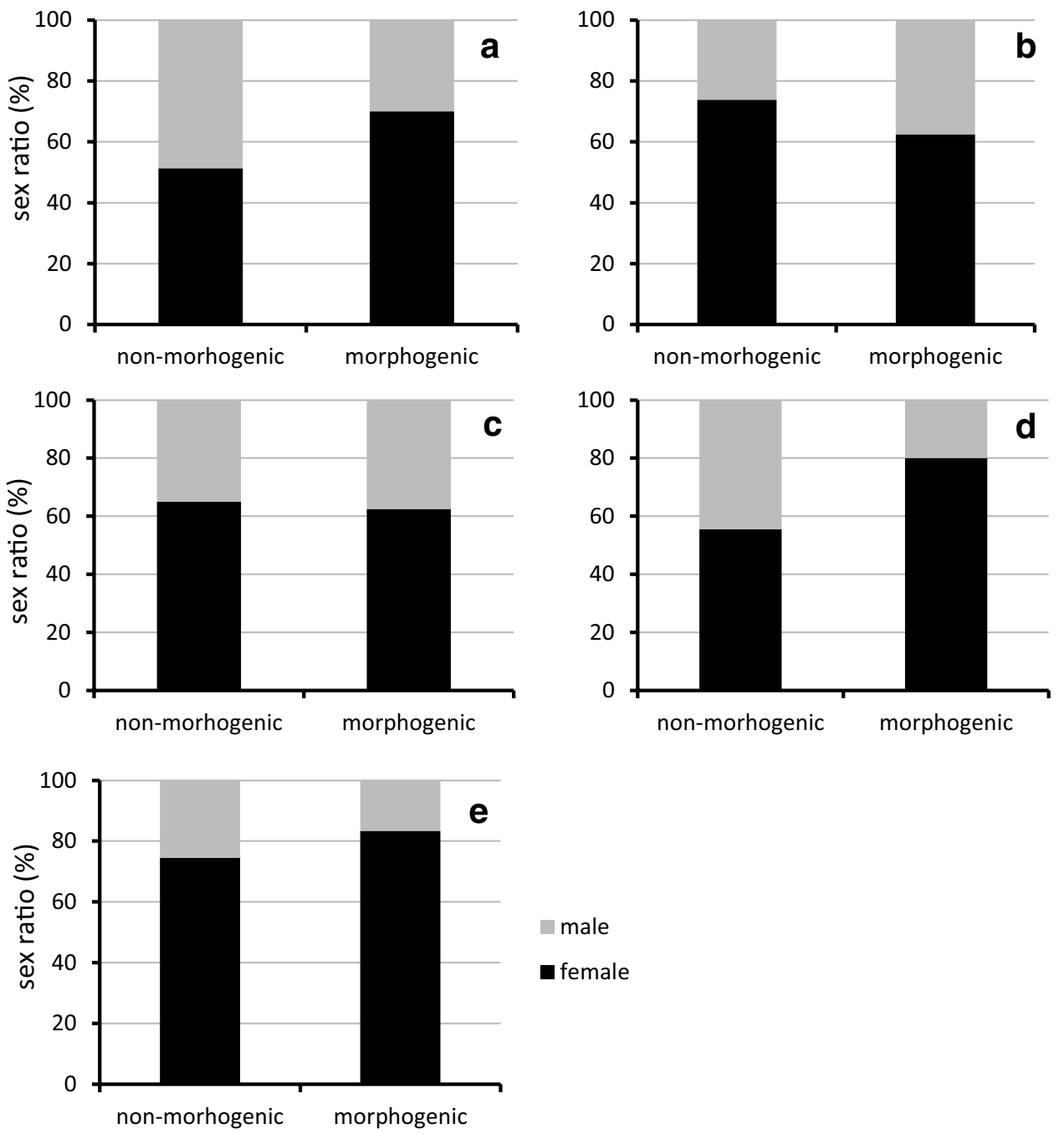

Y chromosomes of $R$. acetosa (Navajas-Peréz et al. 2006), proved to be effective for sexing the germinated seedlings and hypocotyls of $R$. thyrsiflorus.

Biased sex ratios are common in populations of dioecious species and the deviations from equality might be triggered by a variety of mechanisms (Barrett et al. 2010) and may reflect the interactions between sex-based differences in the costs of reproduction, life history, and ecological factors (Field et al. 2013). Stehlik et al. (2008) demonstrated that the local pollination environment can influence the progeny sex ratios in populations of dioecious plants such as $R$. nivalis. The females that were located close to males captured the most pollen, produced the highest number of seeds, and revealed the most female-biased sex ratios. The probable explanation for the relation between higher stigmatic pollen loads and female-biased primary sex ratios is the selective fertilisation as the effect of differential pollentube growth of females versus males.

Field et al. (2013) reported significant heterogeneity in sex ratios among dioecious flowering plants and most of this variation appeared to reflect the type of sampling, the demographic characteristics of the populations, and different response of sexes to environmental stress. They noted that in xeric conditions and at higher altitudes and latitudes a greater male bias in populations could be observed, which illustrated the reduced growth and survival rate of females in more stressful conditions. Petry et al. (2016) documented sex-specific responses to climate change in Valeriana edulis. Increased elevation

Table 2 Results of the generalised linear model with morphogenesis as a dependent variable

\begin{tabular}{lrll}
\hline Parameter & Wald $\chi^{2}$ & df & Significance \\
\hline Intercept & 72.556 & 1 & 0.000 \\
Medium (M) & 3.289 & 4 & 0.511 \\
Sex (S) & 0.932 & 1 & 0.334 \\
$\mathrm{M} \times \mathrm{S}$ & 2.338 & 4 & 0.665 \\
\hline
\end{tabular}

A binomial distribution of the dependent variable is assumed 
resulted in increased water availability and female frequency, which was likely a result of sex-specific efficiency of water use and survival. Chaurasia and Shukla (2016) indicated that the female-biased sex ratio of Trewia nudiflora was probably a response to greater soil moisture and a higher light environment, which ensured the production and maturation of large fleshy fruits with no chance of their being desiccated or hindered in seed dispersal.

According to Stehlik and Barrett (2005), a higher male mortality might be observed in $R$. nivalis under stressful conditions, and in R. thyrsiflorus and R. nivalis the female bias increases during their perennial life cycles (Rychlewski and Zarzycki 1986; Stehlik et al. 2007). Studies on R. nivalis revealed an interesting relation between elevations and sex ratios (Stehlik and Barrett 2005). Although the seed sex ratios were the least biased at low elevations, the sex ratios of adults were strongly female-biased indicating that ecological conditions caused significant male mortality. Contrary, at higher elevations the differences in sex ratios between the seeds and adults were not significant, indicating the decrease in male mortality under more alpine conditions (Stehlik and Barrett 2005).

Ueno et al. (2007) reported that in Salix sachalinensis, the timing of sexual maturation in females and males, particularly in the early life stages, determined the changes in the sex ratio. They observed that males were more numerous than females in young populations, probably as a result of precocious reproduction of males as a result of their lower reproductive costs. The sex ratio changed from malebiased to female-biased by an intermediate age. Pickup and Barrett (2013) stated that female-biased sex ratios in $R$. hastatulus were probably caused by interactions between demographic and genetic factors.

Interesting results were obtained in Aruncus sylvestris in studies on sexual dimorphism in reference to vegetative features and on the sex ratio in different environmental conditions (Marciniuk et al. 2010). It was demonstrated that females were taller, produced longer inflorescences, and had longer and wider leaves than males. In populations growing in optimum ecological conditions (fertile wet soil, half-light) the sex ratio was close to $1: 1$. Changes of ecological conditions along the gradient of fertility, habitat moisture, and intensity of solar radiation caused a marked drift of sex structure towards the male domination. Light intensity was the crucial factor for the sex ratio in studied populations. Those growing in shade and in full light showed a significant shift to male dominance. The largest deviation from the 1:1 sex ratio $(\mathrm{M}: \mathrm{F}=1: 0.2)$ was observed in extremely unfavorable habitat conditions (full sun irradiance, dry soil). Results obtained by Marciniuk et al. (2010) demonstrated that female individuals of Aruncus sylvestris had markedly higher habitat requirements compared with the males.

Eppley (2006) revealed that in Distichlis spicata a variation in the sex ratio could be caused by the differences in competitive abilities at the seedling stage, with female seedlings exhibiting a stronger environment dependent competitive ability than male seedlings. It was suggested that sex-specific competitive differences could have a greater impact on seedlings, and research was needed to explain if such competitive interactions occured at the vulnerable seedling stage.

Stehlik and Barrett (2005) determined that male $R$. nivalis seeds were significantly heavier than female seeds. Glasshouse experiments showed that although the male seeds germinated earlier than the females, female individuals produced their leaves more rapidly and developed more shoots and longer leaves compared to males.

We obtained different results during experiments related to the germination of male and female seeds in Petri dishes under in vitro conditions. A molecular analysis of the germinated seedlings in our study revealed a sex ratio of $\mathrm{M}: \mathrm{F}=1: 2.19$. According to Rychlewski and Zarzycki (1986), the dominance of female seeds might be the effect of for example the higher mortality of male zygotes or embryos, but the differential survival rate appeared to be the factor that influenced mostly the sex ratio in populations of $R$. thyrsiflorus. During our study, the seeds were germinated on moist sterile filter paper under the same conditions of humidity, light, and temperature. The germination of the seeds was measured for 11 days, which was the number of days that elapsed from sowing to the appearance of the roots and cotyledons in the male and female seeds. No differences in the germination time were observed between the male and female seeds when both the appearance of the roots and cotyledons were considered. Our results revealed that there was no competition between the sexes during seed germination when grown in uniform growth conditions.

In our study, the first complete micropropagation protocol from hypocotyls explants was developed for $R$. thyrsiflorus. This procedure can be useful to plant breeders and scientists for clonal propagation, physiological research, and for the analysis of the production of secondary metabolites by male and female explants.

The highest frequency of explants that revealed a morphogenetic response with the highest number of regenerated adventitious shoot buds was observed on MS medium supplemented with $2.27 \mu \mathrm{M}$ TDZ. Histological and SEM analyses showed indirect organogenesis. Secondary organogenesis was also observed. Similar results were obtained by Ślesak et al. (2015) with male adventitious root culture, in which the medium supplemented with $2.27 \mu \mathrm{M}$ TDZ proved to be the best to induce a highly efficient indirect 
morphogenetic response. Thidiazuron was able to increase the accumulation of auxin and cytokinin, and it can act as a substitute for both these plant growth regulators (Murch and Saxena 2001).

In our study, somatic embryogenesis was also obtained, but only on the male hypocotyls cultured on MS medium supplemented with $9.05 \mu \mathrm{M}$ 2,4-D and $8.88 \mu \mathrm{M}$ BA and 5\% sucrose. Plant hormones and/or stress treatments belong to culture conditions able to initiate somatic embryo development. Among plant hormones, auxin and cytokinin, the key regulators of plant cell division and differentiation, are used with success the most frequently, but abscisic acid considered to be a plant "stress hormone" can also be useful. The interaction between the factor inducing somatic embryogenesis and the endogenous hormone levels of the explants is dependent on the explant source. More responsive for induction are explants with higher levels of endogenous auxin. Factors, which induced somatic embryogenesis, may directly or indirectly affect the auxin balance and/ or responses within the cells (Fehér 2015 and literature therein). According to Fraga et al. (2016) cytokinins are also important during the initial phase of somatic embryogenesis, but not for the later stages of embryo development and maturation. The various cell-types exhibit specific responses to the same stress factor, what can explain the striking influence of the genotype, explant source, culture, and stress condition on the embryogenic capability. The following stress conditions used to initiate somatic embryo formation: osmotic stress, heavy metal stress, temperature, hypoxia, ultraviolet radiation, and mechanical or chemical treatments (Fehér 2015 and literature therein). Interesting results were obtained during experiments on elite sugarcane variety. Ferreira et al. (2017) indicated that the induction of somatic embryogenesis depends on the type of light source and should be conducted under fluorescent lighting (FL) contrary to the remaining micropropagation process, which should be performed using a light emitting diode (LED).

Bogunia and Przywara (2000) reported that carbohydrates were biomolecules that were necessary for plant morphogenesis under in vitro conditions. Karami et al. (2006) revealed that increasing the concentration of sucrose enhanced the formation of embryogenic callus and somatic embryos in Dianthus caryophyllus, which confirmed that the role of sucrose may be interpreted as performing both the nutritional and osmotic regulatory functions. Similarly, in our study, a higher sucrose concentration induced the formation of somatic embryos.

Our results of the sex molecular analysis of the hypocotyls cultured in vitro revealed that the efficiency of morphogenesis varied in the sexes and depended on the ratio of auxin and cytokinin in the culture medium. A certain ratio of these plant growth regulators in the medium determined the higher morphogenetic reaction of the female or male explants of $R$. thyrsiflorus.

A higher female response was observed in the medium supplemented with 2,4-D and BA in similar concentration (9.05 $\mu \mathrm{M} 2,4-\mathrm{D}$ and $8.88 \mu \mathrm{M} \mathrm{BA})$ and with the medium to which auxins were added in a two-fold higher concentration (4.52 $\mu \mathrm{M}$ 2,4-D) compared to the cytokinin concentration $(2.22 \mu \mathrm{M}$ BA). The opposite reaction was observed on the medium in which the cytokinin concentration was two-fold higher $(8.88 \mu \mathrm{M} \mathrm{BA})$ compared to the concentration of auxin $(4.52 \mu \mathrm{M} 2,4-\mathrm{D})$. In this case, the decrease in the participation of female explants among morphogenetic explants was evident. Based on these results, we can suppose that the different levels of endogenous growth regulators in male and female hypocotyls could determine their ability to undergo morphogenesis on different culture medium.

Experiments to investigate the physiological differences (level of endogenous growth regulators, proteins related to stress responses, and antioxidant enzymes) in male and female explants are in progress. The initial results which revealed differences in the superoxide dismutase isoforms in the explants of different sexes are encouraging.

\section{Conclusions}

We developed a procedure for the micropropagation of $R$. thyrsiflorus from hypocotyls explants, which can be useful for analyses of a sex-specific morphogenetic reaction under in vitro conditions. The molecular analysis performed, based on genetic sex markers, allowed the sex of the explants to be determined in order to create an in vitro culture of male or female explants. The relationship between morphogenetic response and the sex of explants cultured under in vitro conditions was analysed. Based on the results, we can conclude that regeneration from hypocotyls of $R$. thyrsiflorus primarily occurred via indirect adventitious shoot formation and also via somatic embryogenesis, which was confirmed by histological and SEM analyses. The highest frequency of explants that revealed a morphogenetic response with the highest number of regenerated adventitious shoot buds was observed on the MS medium that had been supplemented with $2.27 \mu \mathrm{M}$ TDZ. The efficiency of morphogenesis varied in the different sexes and depended on the ratio of auxin and cytokinin in the culture medium. No differences in germination time between male and female seeds and in terms of male and female seedling length were observed, which indicates that there is no competition between the sexes during seed germination when they are growing under uniform growth conditions. 
Acknowledgements The work was partially supported by the statutory research funds DS/MND/WBiNoZ/IB/11/2014 K/DSC/002293 of the Department of Plant Cytology and Embryology, Jagiellonian University in Cracow. We would like to thank Labsoft-K. Herman (Leica Microsystems) for the possibility to make SEM images.

Open Access This article is distributed under the terms of the Creative Commons Attribution 4.0 International License (http:// creativecommons.org/licenses/by/4.0/), which permits unrestricted use, distribution, and reproduction in any medium, provided you give appropriate credit to the original author(s) and the source, provide a link to the Creative Commons license, and indicate if changes were made.

\section{References}

Barrett SCH, Yakimowski SB, Field DL, Pickup M (2010) Ecological genetics of sex ratios in plant populations. Philos T R Soc B 365:2549-2557

Błocka-Wandas M, Sliwinska E, Grabowska-Joachimiak A, Musiał K, Joachimiak AJ (2007) Male gametophyte development and two different DNA classes of pollen grains in Rumex acetosa L., a plant with an $\mathrm{XX} / \mathrm{XY}_{1} \mathrm{Y}_{2}$ sex chromosome system and femalebiased sex ratio. Sex Plant Reprod 20:171-180

Bogunia H, Przywara L (2000) Effect of carbohydrates on callus induction and regeneration ability in Brassica napus L. Acta Biol Cracov Bot 42:79-86

Chaurasia B, Shukla RP (2016) Changes in reproductive phenology and sex ratio of Trewia nudiflora Linn. growing in sal forest of north-eastern Uttar Pradesh, India. Trop Ecol 57(1):89-99

Eppley SM (2006) Females make tough neighbors: sex-specific competitive effects in seedlings of a dioecious grass. Oecologia 146:549-554

Fehér A (2015) Somatic embryogenesis-stress-induced remodeling of plant cell fate. Biochim Biophys Acta 1849:385-402

Ferreira LT, Silva MMA, Ulisses C, Camara TR, Willadino L (2017) Using LED lighting in somatic embryogenesis and micropropagation of an elite sugarcane variety and its effect on redox metabolism during acclimatization. Plant Cell Tiss Organ Cult 128:211-221

Field DL, Pickup M, Barrett SCH (2012) Comparative analyses of sex-ratio variation in dioecious flowering plants. Evol Int J Org Evol 67(3):661-672

Field D, Pickup M, Barrett SCH (2013) Ecological context and metapopulation dynamics affect sex-ratio variation among dioecious plant populations. Ann Bot-London 111:917-923

Fraga HPF, Vieira LN, Puttkammer CC, Santos HP, Garighan JA, Guerra MP (2016) Glutathione and abscisic acid supplementation influences somatic embryo maturation and hormone endogenous levels during somatic embryogenesis in Podocarpus lambertii Klotzsch ex Endl. Plant Sci 253:98-106

Gawal NJ, Jarret RL (1991) A modified CTAB DNA extraction procedure for Musa and Ipomoea. Plant Mol Biol Rep 9:262-266

Grabowska-Joachimiak A, Kwolek D, Kula A, Marciniuk P (2012) Fluorescent banding pattern and species-specific DNA marker in Rumex thyrsiflorus Fingerh. Cytogenet Genome Res 137:70-77

Hardy ICW (2002) Sex ratios: concepts and research methods. Cambridge University Press, Cambridge

IBM Corp (2016) IBM SPSS Statistics for Windows, Version 24. IBM Corp., Armonk, NY

Karami O, Deljou A, Esna-Ashari M, Ostad-Ahmadi P (2006) Effect of sucrose concentrations on somatic embryogenesis in carnation (Dianthus caryophyllus L.). Sci Hortic 110:340-344
Korpelainen H (2002) A genetic method to resolve gender complements investigations on sex ratios in Rumex acetosa. Mol Ecol 11:2151-2156

Kwolek D, Joachimiak AJ (2011) Seed sexing revealed female bias in two Rumex species. Acta Soc Bot Pol 80(2):93-97

Lajter I, Zupkó I, Molnár J, Jakab G, Balogh L, Vasas A, Hohmann J (2013) Antiproliferative activity of Polygonaceae species from the carpathian basin against human cancer cell lines. Phytother Res 27:77-85

Litvinenko Y, Muzychkina RA (2008) New antioxidant phytopreparation from Rumex thyrsiflorus roots. Chem Nat Compd 44(2):239-240

Lloyd DG (1974) Female predominant sex ratios in Angiosperms. Heredity 32:34-44

Marciniuk J, Marciniuk P, Rymuza K (2010) Effects of site conditions on sexual dimorphism and sex ratio in lowland populations of Aruncus sylvestris Kostel. (Rosaceae). Pol J Ecol 58(2):249-257

Mariotti B, Manzano S, Kejnovsky E, Vyskot B, Jamilena M (2009) Accumulation of Y-specific satellite DNAs during the evolution of Rumex acetosa sex chromosomes. Mol Genet Genomics 281:249-259

Murashige T, Skoog F (1962) A revised medium for rapid growth and bioassays with tobacco tissue culture. Physiol Plant 15:437-497

Murch SJ, Saxena PK (2001) Molecular fate of thidiazuron and its effects on auxin transport in hypocotyls tissues of Pelargonium hortorum Bailey. Plant Growth Regul 35:269-275

Navajas-Peréz R, De La Herran R, Jamilena M, Lozano R, Ruiz Rejón M, Garrido-Ramos MA (2005) Reduced rates of sequence evolution of Y-linked satellite DNA in Rumex (Polygonaceae). J Mol Evol 60:391-399

Navajas-Peréz R, Schwarzacher T, De La Herran R, Ruiz Rejón C, Ruiz Rejón M, Garrido-Ramos MA (2006) The origin and evolution of the variability in a Y-specific satellite-DNA of Rumex acetos $a$ and its relatives. Gene 368:61-71

Obeso JR (2002) The costs of reproduction in plants. New Phytol 155:321-348

Orbán-Gyapai O, Lajter I, Hohmann J, Jakab G, Vasas A (2015) Xanthine oxidase inhibitory activity of extracts prepared from Polygonaceae species. Phytother Res 29:459-465

Petry WK, Soule JD, Iler AM, Chicas-Mosier A, Inouye DW, Miller TEX, Mooney KA (2016) Sex-specific responses to climate change in plants alter population sex ratio and performance. Science 353(6294):69-71

Pickup M, Barrett SCH (2013) The influence of demography and local mating environment on sex ratios in a wind-pollinated dioecious plant. Ecol Evol 3(3):629-639

Rychlewski J, Zarzycki K (1975) Sex ratio in seeds of Rumex acetosa L. as a result of sparse or abundant pollination. Acta Biol Cracov Bot 18:101-113

Rychlewski J, Zarzycki K (1986) Genetical and ecological mechanisms regulating the sex ratio in populations of Rumex thyrsiforus Fingerh. (Polygonaceae). Bull Geobot Inst ETH 87:132-140

Ślesak H, Liszniańska M, Popielarska-Konieczna M, Góralski G, Sliwińska E, Joachimiak AJ (2014) Micropropagation protocol for the hybrid sorrel Rumex tianschanicus x Rumex patientia, an energy plant. Histological, SEM and flow cytometric analyses. Ind Crops Prod 62:156-165

Ślesak H, Góralski G, Kwolek D, Dziedzic K, Grabowska-Joachimiak A (2015) Male adventitious roots of Rumex thyrsiflorus Fingerh. as a source of genetically stable micropropagated plantlets. Plant Cell Tiss Organ Cult 123:193-203

Stehlik I, Barrett SCH (2005) Mechanisms governing sex-ratio variation in dioecious Rumex nivalis. Evol Int $\mathrm{J}$ Org Evol 59(4):814-825 
Stehlik I, Kron P, Barrett SCH, Husband BC (2007) Sexing pollen reveals female bias in a dioecious plant. New Phytol 175:185-194

Stehlik I, Friedman J, Barrett SCH (2008) Environmental influence on primary sex ratio in a dioecious plant. PNAS 105(31):10847-10852

Ueno N, Kanno H, Seiwa S (2006) Sexual differences in shoot production and leaf dynamics in a dioecious tree, Salix sachalinensis. Can J Botany 84:1852-1859
Ueno N, Suyama Y, Seiwa K (2007) What makes the sex ratio female-biased in the dioecious tree Salix sachalinensis? J Ecol 95:951-959

Żuk J (1963) An investigation on polyploidy and sex-determination within the genus Rumex. Acta Soc Bot Pol 32:5-72 